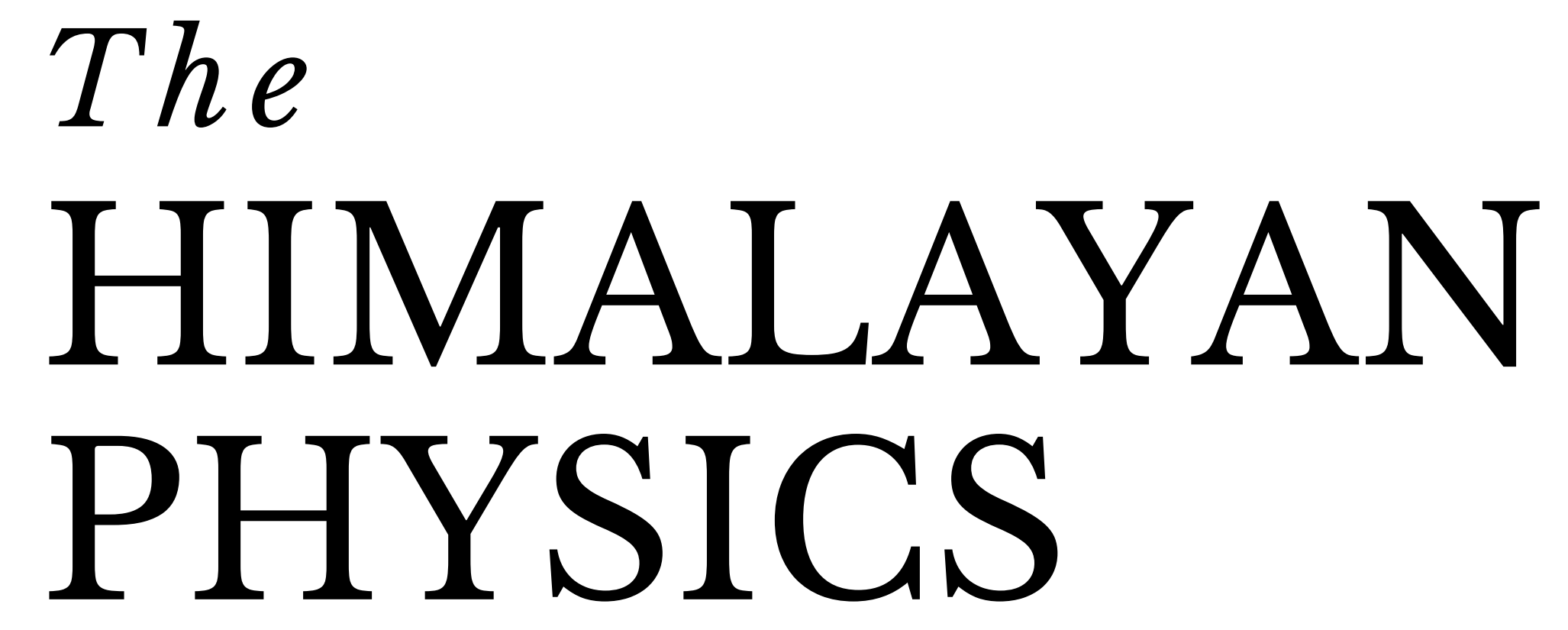

A peer-reviewed Journal of Physics

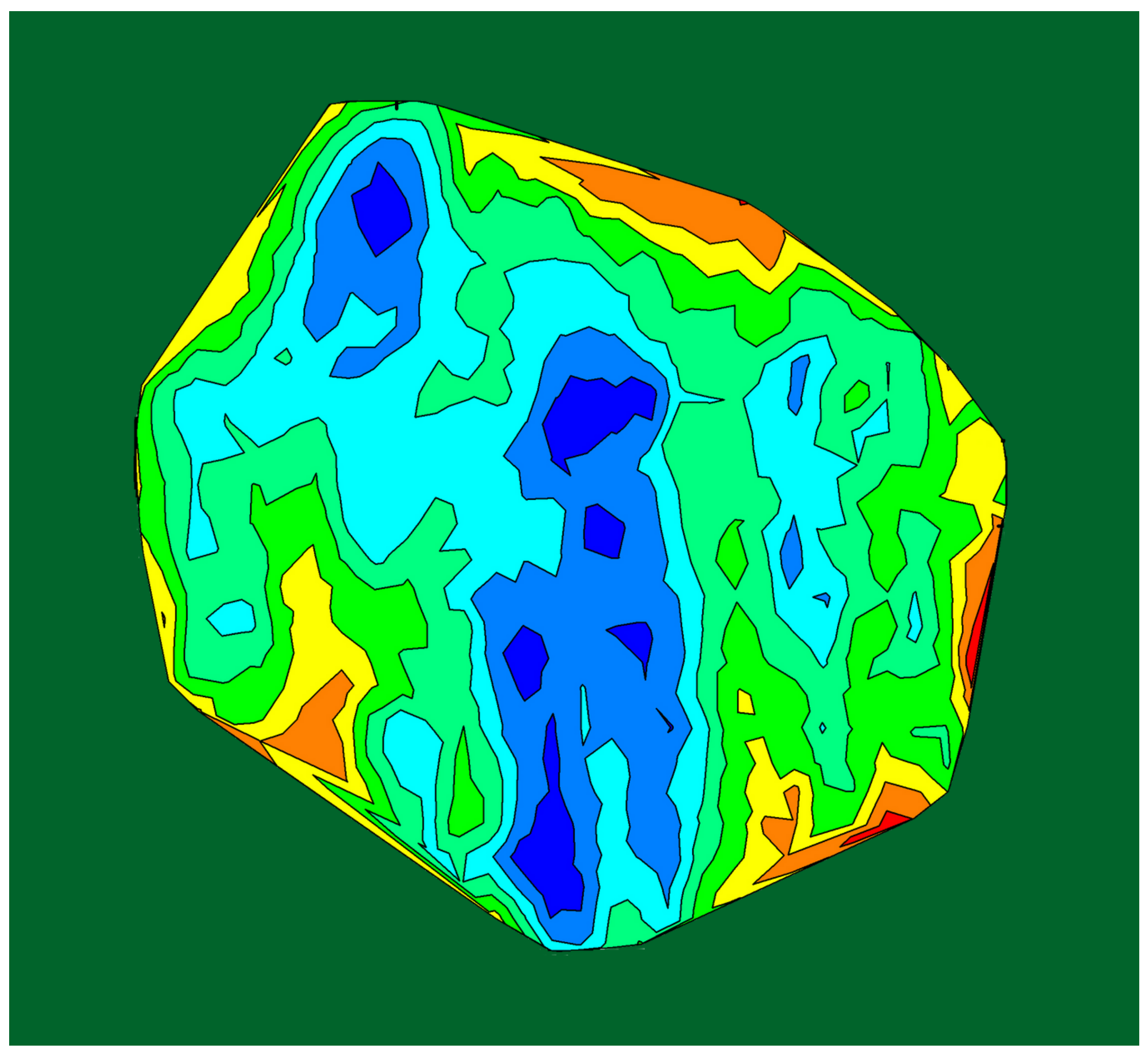

Department of Physics, Prithvi Narayan Campus, Pokhara Nepal Physical Society, Western Chapter, Pokhara 


\section{Publisher}

Department of Physics, Prithvinarayan Campus, Pokhara Nepal Physical Society, Western Chapter, Pokhara

\section{The Himalayan Physics}

Volume 9, December 2020

ISSN 2542-2545

The Himalayan Physics (HimPhys) is an open access peer-reviewed journal that publishes quality articles which make innovative contributions in all areas of Physics. HimPhys is published annually by Nepal Physical Society (Western Regional Chapter), and Department of Physics, Prithvi Narayan Campus, Pokhara. The goal of this journal is to bring together researchers and practitioners from academia in Nepal and abroad to focus on advanced techniques and explore new avenues in all areas of physical sciences and establishing new collaborations with physics community in Nepal.

\section{Chief Editor}

Kapil Adhikari

\section{Associate Editor \\ Aabiskar Bhusal}

(C)2020, Publishers. All rights reserved.

This publication is in copyright. Subject to statutory exception and to the provisions of relevant collective licensing agreements, no reproduction of any part may take place without written permission of the publishers.

Cover: Contour map of dust mass. (C) Mijas Tiwari. Printed from article in the current issue, with permission. 


\section{The HIMALAYAN PHYSICS}

A peer-reviewed Journal of Physics

\section{Chief Editor}

Kapil Adhikari

Associate Editor

Aabiskar Bhusal

\section{Publisher}

Department of Physics, Prithvi Narayan Campus, Pokhara Nepal Physical Society, Western Chapter, Pokhara 


\section{Nepal Physical Society Western Regional Chapter Pokhara, Nepal}

\section{President}

Min Raj Lamsal

Immediate Past President

Jeevan Regmi

Vice-President

Sundar Prasad Dhakal

Secretary

Ravi Karki

Treasurer

Dipak Adhikari

Joint Secretary

Sujan Lamsal

Editorial Member

Kapil Adhikari

Members

Amrit Dhakal

Laxman Thapa

Laxman Timilsina

Narayan Prasad Bhandari

Pradeep Subedi

Advisory Board

Prof. Dr. Pradip K. Bhattarai

Pabitra Mani Poudyal

Surya Bahadur G.C.

Parashu Ram Poudel

Prof. Dr. Shovakanta Lamichhane

Kul Prasad Dahal

Dr. Krishna Raj Adhikari

Ram Sajile Verma 


\section{Himalayan Physics Vol-9 (2020)}

\section{TABLE OF CONTENTS}

Metal Organic Frameworks(MOFs) as efficient carrier for targeted nanodrug delivery

R. Karki, D. Adhikari, K. Adhikari, N. Pantha

A Density Functional Theory Study on Paracetamol-Oxalic Acid Co-Crystal

P. Paudel, K.R. Adhikari, K. Adhikari

First-principles study of $\mathrm{C}$ cites vacancy defects in water adsorbed graphene

H.K. Neupane, N.P. Adhikari

Diusion of fructose in water: a molecular dynamics study

S. Bhusal, N. Pantha

Study of aecting factors of meteorological parameters on solar radiation on Pokhara P.M. Shrestha, J. Regmi, U. Joshi, K.N. Poudyal, N.P. Chapagain, I.B. Karki

Variation of mean value of velocity of ion with dierent obliqueness of magnetized plasma sheath

B.R. Adhikari, H.P. Lamichhane, R. Khanal

Study of dust properties of two far infrared cavities nearby asymptotic giant branch stars under infrared astronomical satellite maps

M. Tiwari, S.P. Gautam, A. Silwal, S. Subedi, A. Paudel, A. K. Jha

An experimental study on irradiated interface of silicon

M.R. Lamsal

Calculation of energy loss of proton beam on thyroid tumor

K. Giri, B. Paudel, B.R. Gautam

Study of noise level status at dierent rice mills in Surkhet Valley, Nepal

D.R. Paudel, H.N. Baral

Elliptically polarized laser assisted elastic electron-hydrogen atom collision and differential scattering cross-section

K. Yadav, S.P. Gupta, J.J. Nakarmi

Geodynamics of Gorkha earthquake (Mw 7.9) and its aftershocks

R.K. Tiwari and H. Paudyal 


\title{
Diffusion of fructose in water: a molecular dynamics study
}

Research Article

\author{
Santosh Bhusal ${ }^{1 *}$, Nurapati Pantha ${ }^{2}$ \\ 1 GoldenGate International College, Tribhuvan University, Battisputali, Kathmandu, Nepal \\ 2 Central Department of Physics, Tribhuvan University, Kirtipur, Kathmandu, Nepal
}

\begin{abstract}
Present work carries the molecular dynamics (MD) simulation to study the self-diffusion coefficients of fructose $\left(\mathrm{C}_{6} \mathrm{H}_{12} \mathrm{O}_{6}\right)$ and SPC/E (Extended Simple Point Charge) water $\left(\mathrm{H}_{2} \mathrm{O}\right)$ along with their binary diffusion coefficients at different temperature $(298.15 \mathrm{~K}, 303.15 \mathrm{~K}, 308.15 \mathrm{~K}$ and $312.15 \mathrm{~K})$. A dilute solution of 3 molecules of fructose (solute, mole fraction 0.0018) and 1624 molecules of water (solvent, mole fraction 0.9982) has been taken for making it relevent to the previously reported experiment. The structural analysis of the mixture has been estimated by using the radial distribution function (RDF) of its constituents. Mean square displacement (MSD) and Einstein's relation have been used to find the self-diffusion coefficients of both the solute and solvent. Furthermore, Darken's relation finds the binary diffusion coefficients. The temperature dependence of diffusion coefficients follows the Arrhenius behaviour which further calculates activation energy of diffusion. The results from the present work agree well with the previously reported experimental values.

Keywords: Fructose • Diffusion coefficient • Molecular dynamics • RDF • Arrhenius behavior
\end{abstract}

\section{Introduction}

Fructose is a simple sugar (monosaccharides) found in many foods and one of the three most important blood sugars along with glucose and galactose[1]. Fructose is a monosaccharide isomer with the same empirical formula as glucose $\left(\mathrm{C}_{6} \mathrm{H}_{12} \mathrm{O}_{6}\right)$ but with different structure[1].

Its IUPAC name is 1,3,4,5,6 - Pentahydroxy - 2 - hexanone and chemical formula is $\mathrm{C}_{6} \mathrm{H}_{12} \mathrm{O}_{6}[2]$. Fructose is the sweetest among all other sugar[2]. Fructose is odorless and colorless substance found in white, crystalline or granular powder form[3]. The fructose has a molar mass of $180.159 \mathrm{~mol} / \mathrm{g}$ and density in solid crystalline form is $1.60 \mathrm{~g} / \mathrm{cm}^{3}$ [4]. It has melting point of $103^{\circ} \mathrm{C}[5]$. Fructose has higher solubility than other sugar and refined crystallised fructose appears white in color. Fructose produces clear colorless liquid in water[6] and has a solubility of $4 \mathrm{~g}$ per gram $\mathrm{H}_{2} \mathrm{O}$ at $25^{\circ} \mathrm{C}[4]$.

Fructose has been a part of the human diet for many thousand of years which is found in highest concentrations in fruits and to a lesser degree in vegetables[7]. Basically, fructose is the fruit sugar and more

\footnotetext{
* Corresponding Author: protonsantosh@gmail.com
} 
sweeter than glucose and sucrose. Cane, beet and corn sugars are produced industrially and their use result in significant quantities of added sugar in our diet, about half of which is fructose[7]. It is commonly used as sweetening agent along with glucose under the name High Fructose Corn Syrup (HFCS) for soft drinks. Cells process fructose to extract energy through a process called aerobic respiration, which essentially means burning of fructose in presence of oxygen to produce ATP, the cellular energy molecule. Hence, like glucose, fructose is a source of energy for the cells[8]. The fructose is stored as glycogen by liver cell which can be later broken down by liver to release glucose into the blood stream for use by all body cells[8]. Thus, fructose also helps to maintain blood sugar level. Fructose is essential for spermatozoa metabolism and spermatozoa mobility[9]. Fructose, in semen, is the source of energy of every sperm activites[10]. Thus, fructose is one of the important monosaccharide for living organism. The intake of soft drinks containing High Fructose Corn Syrup (HFCS) can cause obesity. The dietary fructose is potential risk factor for cardiovascular diseases. Study shows that eating diet high in fructose consistently causes hyperlipidemia in rodents, dogs and non-human primates[11]. Excessive consumption of fructose leads to insulin resistance, obesity, cardiovascular diseases etc[12].

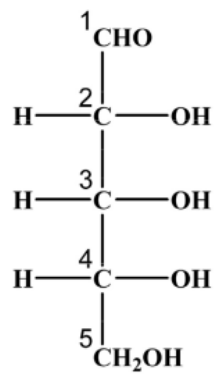

Ribose

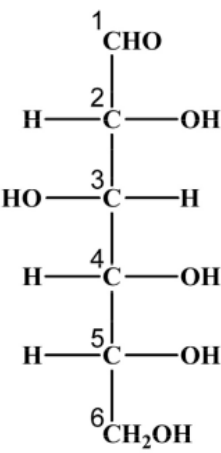

Glucose

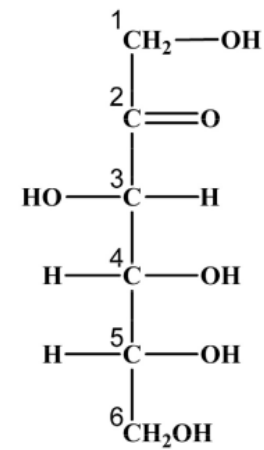

Fructose

Figure 1. Structure of monosaccharides [13].

The computer simulation is the ever growing virtual method for observing the interacting particles. This method is used widely everywhere in the world due to its cost effectiveness. Computer simulation has vital role in providing the exact results for many problems in statistical method which were only soluble by approximate method. The strongest aspect of computer simulation is that its result can be compared and analyzed with experimental results by bridging various analogous method. There are two often used computer simulation techniques : Monte Carlo method and Molecular Dynamics (MD) simulation[14]. Monte Carlo method is widely used for structural representations of molecule such as hardspheres and disks whereas Molecular Dynamics is used to explain the solution of classical equation of motion (Newton's Equation). MD describes structural as well as time evolution of the system[14]. The MD simulation is used to study the transport properties of various biomolecules, structure and dynamics of protein, protein folding, docking of protein, first and second order phase transition etc[15]. There are wide range of usages of MD simulation. 
Diffusion is the process in which matter is transported from one part to another due to random molecular motion[16]. It is one of the most important biochemical phenomena to occur in nature. It is responsible for transport and exchange of important gases, minerals and nutrients in all living beings. Diffusion process is important for the kinetics of many microstructural changes that occur during preparation, processing and heat treatment of materials which include nucleation of new phases, diffusive phase transformations, homogenization of alloys, recrystallization etc[17]. The applications of diffusion include prcocess such as doping during fabrication of microelectronic devices, operation of solid electrolytes for batteries and fuel cells, surface hardening of steel through carburization or nitridation, diffusion bonding and sintering[17].

The first attempt to measure self-diffusion (the most basic diffusion process) was that of physico-chemist Georg Karl Von Hevesy who studied self diffusion in liquid and in solid lead by using a natural radioisotope ${ }^{210} \mathrm{~Pb}$ and ${ }^{212} \mathrm{~Pb}$ of lead. From the pioneering work of Alder and Wainwright the simulation of diffusion coefficient has been an area of continuous research. The equations of Fick, the statistical interpretation of diffusion coefficient by Einstein and Smoluchowski and the Boltzmann-Matano method for concentration dependent diffusion coefficients opened the way for experimental techniques[18].

After the introduction part, we describe about the theory of diffusion, modeling of system and simulation procedure, results and dicussion, and conlcusions in order.

\section{Methodology}

\section{Diffusion Coefficient}

Diffusion is a transport phenomenon which occurs due to the presence of concentration gradient[19]. The transport of mass takes place from higher chemical potential region to the lower chemical potential region till the steady state is achieved[20]. Diffusion is a response property of the system to a concentration inhomogeneity[21]. Self-diffusion is the diffusion in absence of a chemical potential gradient in homogeneous medium and corresponding diffusion coefficient is called self-diffusion coefficient[22]. The self-diffusion coefficient is usually calculated by Einstein's relation using mean square displacement (MSD) plot against time (t) and by Green-Kubo's relation using velocity autocorrelation function (VACF). The Einstein's relation to calculate self-diffusion coefficient is given by[23]

$$
D=\lim _{t \rightarrow \infty} \frac{\left\langle\mathbf{r}^{2}(t)\right\rangle}{6 t}
$$

where $D$ is self-diffusion coefficient, $\left\langle\mathbf{r}^{2}(t)\right\rangle$ is the mean square displacement and $t$ is time.

The Einstein's relation for 3-D system is given as[14, 21]

$$
D=\lim _{t \rightarrow \infty} \frac{\left\langle\left[\mathbf{r}_{\alpha}\left(t+t_{0}\right)-\mathbf{r}_{\alpha}\left(t_{0}\right)\right]^{2}\right\rangle}{6 t}
$$

where $\alpha$ denotes the type of component (solute or solvent) and $t_{0}$ is any time origin. The angled brackets $\langle\ldots\rangle$ indicate the ensemble average. The ensemble average is taken over all atoms of the component $\alpha$ in the simulation 
and all time origins $[22,24]$.

In the present work, we calculate the self-diffusion coefficients of fructose (solute) and water (solvent) using Einstein's relation and thus, calculated self-diffusion coefficients are used to estimate binary diffusion coefficient using Darken's relation[25]

$$
\mathrm{D}_{12}=\mathrm{N}_{2} \mathrm{D}_{1}+\mathrm{N}_{1} \mathrm{D}_{2}
$$

where $\mathrm{D}_{12}$ is binary diffusion coefficient, $\mathrm{D}_{1}$ and $\mathrm{D}_{2}$ are the self-diffusion coefficients of molecules 1 and 2 respectively and $\mathrm{N}_{1}$ and $\mathrm{N}_{2}$ are the corresponding mole fractions.

\section{Modeling of the system}

We have carried out molecular dynamics simulation in GROningen MAchine for Chemical Simulation (GROMACS) 5.1.1 package using OPLS/AA force field. The system consists of 3 molecules of fructose and 1624 SPC/E water molecules. The simulation has been carried out at four temperatures i.e. 298.15 K, $303.15 \mathrm{~K}$, $308.15 \mathrm{~K}$ and $312.15 \mathrm{~K}$. The total potential energy $\left(U_{\text {total }}\right)$ of the system comprises of the contribution by both bonded and non-bonded interactions. The bonded interaction consists bond stretching $\left(U_{b}\right)$, bond-angle bending $\left(U_{a}\right)$, bond dihedral $\left(U_{d}\right)$ and out of plane distortion $\left(U_{i d}\right)$ potential. The non-bonded interactions are given by Van der Waals potential or Lennard-Jones Potential $\left(U_{L J}\right)$ and coulomb potential $\left(U_{c}\right)$. Now, the total potential energy is given as[26]

$$
U_{\text {total }}=U_{b}+U_{a}+U_{d}+U_{i d}+U_{L J}+U_{c}
$$

The harmonic potential represents the bond stretching between two covalently bonded atoms $i$ and $j$, such that bond stretching potential is given by[26]

$$
U_{b}\left(r_{i j}\right)=\frac{1}{2} K_{i j}^{b}\left(r_{i j}-b_{i j}\right)^{2}
$$

where $\mathrm{K}_{i j}^{b}$ is the force constant and $\mathrm{b}_{i j}$ is the equilibrium bond length between $i$ and $j$ atoms. The bond-angle vibration between a triplet of atoms $i-j-k$ is represented by a harmonic potential on the angle $\Theta_{i j k}$ [26]

$$
U_{a}\left(\Theta_{i j k}\right)=\frac{1}{2} K_{i j k}^{\Theta}\left(\Theta_{i j k}-\Theta_{i j k}^{0}\right)^{2}
$$

where the force constant is given by $K_{i j k}^{\Theta}$, angle between atoms is $\Theta_{i j k}$ and $\Theta_{i j k}^{o}$ is the equilibrium bond angle.

Proper dihedral angle is defined by dihedral angle $(\phi)$ between ijk and jkl planes which involves four atoms for formation of the planes. The periodic dihedral potential is given as [26]

$$
U_{p d}\left(\phi_{i j k l}\right)=k_{i j k l}^{c}\left(1+\cos \left(n_{i j k l} \phi_{i j k l}-\phi_{o}\right)\right)
$$

where $k_{i j k l}^{c}$ is the force constant, $\phi_{i j k l}$ is proper dihedral angle, $\phi_{o}$ is the angle where potential passes minimum value and $n_{i j k l}$ is multiplicity. The proper dihedral : Ryckaert-Bellemans potential function is given by [26]

$$
U_{R B}\left(\phi_{i j k l}\right)=\sum_{n=0}^{5} C_{n}(\cos (\psi))^{n}
$$


where $\psi=\phi-\pi^{c} ; \phi$ is dihedral angle and $C_{0} \ldots . . C_{5}$ are Ryckaert- Bellemans parameters.

The nonbonded interatomic interaction is the sum of Lennard-Jones interaction $\left(U_{L J}\right)$ and coulomb interaction $\left(U_{c}\right)$ and given as $[26]$

$$
\begin{gathered}
U\left(r_{i j}\right)=U_{L J}\left(r_{i j}\right)+U_{c}\left(r_{i j}\right) \\
U\left(r_{i j}\right)=4 \epsilon_{i j}\left[\left(\frac{\sigma_{i j}}{r_{i j}}\right)^{12}-\left(\frac{\sigma_{i j}}{r_{i j}}\right)^{6}\right]+\frac{q_{i} q_{j}}{4 \pi \epsilon_{o} r_{i j}}
\end{gathered}
$$

where $\sigma_{i j}=\frac{1}{2}\left(\sigma_{i i}+\sigma_{j j}\right), \epsilon_{i j}=\left(\epsilon_{i i} \epsilon_{j j}\right)^{\frac{1}{2}}$ and $r_{i j}$ is the cartesian distance between two atoms $i$ and $j$.

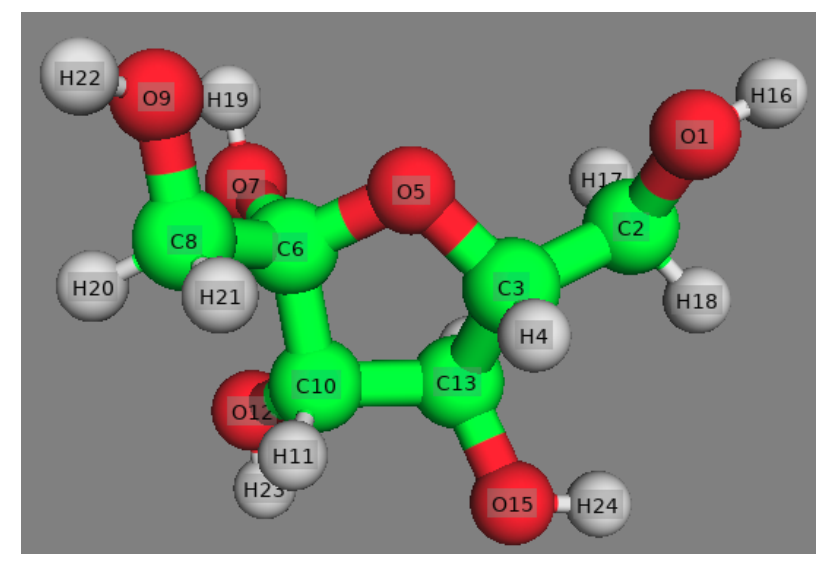

Figure 2. Structure of fructose molecule obtained from FRU.pdb file[27].

The bonded parameters for SPC/E water is given in the Table 1.

Table 1. Force-field (bonded) parameters for SPC/E water.

\begin{tabular}{cccc}
\hline$K_{\mathrm{OH}}\left(\mathrm{kJmol}^{-1} \mathrm{~nm}^{-2}\right)$ & $\mathrm{b}_{\mathrm{OH}}(\mathrm{nm})$ & $K_{\mathrm{HOH}}\left(\mathrm{kJmol}^{-1} \mathrm{rad}^{-2}\right)$ & $\Theta_{o}$ \\
\hline $3.4500 \times 10^{5}$ & 0.1 & $3.8300 \times 10^{2}$ & $109.47^{\circ}$ \\
\hline
\end{tabular}

In Table $1, b_{\mathrm{OH}}$ is the equilibrium bond length between oxygen and hydrogen atoms $(\mathrm{O}-\mathrm{H})$ in water molecule. $K_{\mathrm{OH}}$ is the force constants of the bonds $\mathrm{O}-\mathrm{H}$ in water molecule. Similarly, $\Theta_{0}$ is the equilibrium bond angle (HOH) and $K_{\mathrm{HOH}}$ is the strength of the bond angle vibration potential in water molecules.

The non-bonded parameters for oxygen (O9) atom of fructose and water is given in the Table 2.

Table 2. OPLS/AA force-field (non-bonded) parameters for oxygen (O9) atom of fructose and SPC/E water.

\begin{tabular}{cccc}
\hline O9 & Values & Water & Values \\
\hline$\sigma_{\mathrm{O} 9-\mathrm{O} 9}$ & $0.3070 \mathrm{~nm}$ & $\sigma_{\mathrm{OW}-\mathrm{OW}}$ & $0.3165 \mathrm{~nm}$ \\
$\epsilon_{\mathrm{O} 9-\mathrm{O} 9}$ & $85.55 \mathrm{k}_{B}$ & $\epsilon_{\mathrm{OW}-\mathrm{OW}}$ & $78.2 \mathrm{k}_{B}$ \\
\hline
\end{tabular}

The subscripts O9-O9 and OW-OW represent the oxygen-oxygen interaction in fructose molecules and oxygen-oxygen interaction in water molecules respectively. The parameters for SPC/E water presented in the Table 2 are inherent in GROMACS package. The above parameters in Table 2 are for the Lennard-Jones interaction. 
The Coulomb interaction in water molecule arises due to the partial charge of hydrogen atom and oxygen atom which have the values +0.4238 e and -0.8476 e respectively [26]. Similarly, the Coulomb interaction for fructose molecule arises due to the partial charge of carbon, hydrogen and oxygen atoms, where e is the elementary (basic unit) charge.
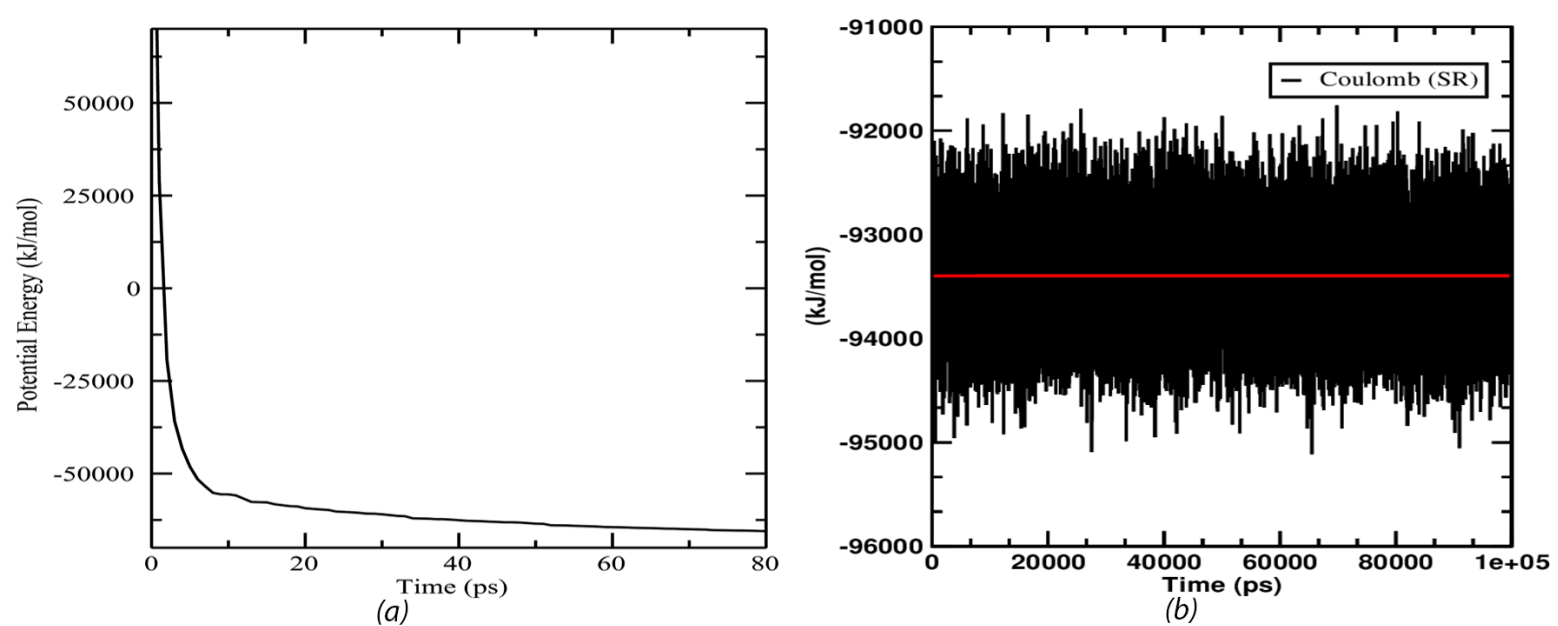

Figure 3. (a) Potential energy as a function of time after energy minimization, (b) Coulomb (short-ranged) potential energy at $298.15 \mathrm{~K}$.

\section{Simulation Procedure}

The molecular dynamics (MD) simulation for binary mixture of fructose and SPC/E water was carried out in a cubical box of size $3.7 \mathrm{~nm}$ with periodic boundary conditions[14] using GROMACS package and OPLS/AA forcefield. The system was subjected to energy minimization with cut-off restriction of $1 \mathrm{~nm}$ to avoid Van der Waals contact caused by the atoms that are too close[18, 22]. The steepest-descent algorithm was used for energy minimization and algorithm stops when the maximum force is less than energy minimization tolerance[26]. The negative potential energy in calculations confirms the energy minimization and local minima of our system. The potential energy of the system after minimization is shown in Fig. 3(a).

The system needs to be in equilibrium state before production run in order to study the dynamical properties. The dynamical variables change with various parameters like temperature, density and pressure. Thus, the system was equilibrated at four different temperatures of $298.15 \mathrm{~K}, 303.15 \mathrm{~K}, 308.15 \mathrm{~K}$ and $312.15 \mathrm{~K}$ and pressure of 1 bar using NPT ensemble to attain the thermal equilibrium. The velocity-rescaling (modified Berendsen) thermostat and Berendsen barostat[26] were used to maintain constant temperature and pressure at coupling time $\tau_{t}=0.01 \mathrm{ps}$ and $\tau_{p}=0.8 \mathrm{ps}$ respectively. The isothermal compressibility of water was taken to be 4.6 $\times 10^{-5} \mathrm{bar}^{-1}$. The system was equilibrated for $100 \mathrm{~ns}$ with the time steps of 2 fs (0.002 ns) using leap-frog integrator. The initial velocity was generated according to Maxwell-Boltzmann distribution function at specified temperature[26]. All the bonds are converted to constraint using LINCS algorithm[26]. The Particle Mesh Ewald (PME) algorithm was used for long range interaction. The cut-off parameter of $1 \mathrm{~nm}$ was considered with periodic 
boundary conditions for coulomb and Lennard-Jones (LJ) interaction[14].

The structure of the system after equilibration is shown in Fig. 4(a). The density and simulated temperatures at different coupling temperatures is shown in Table 3.

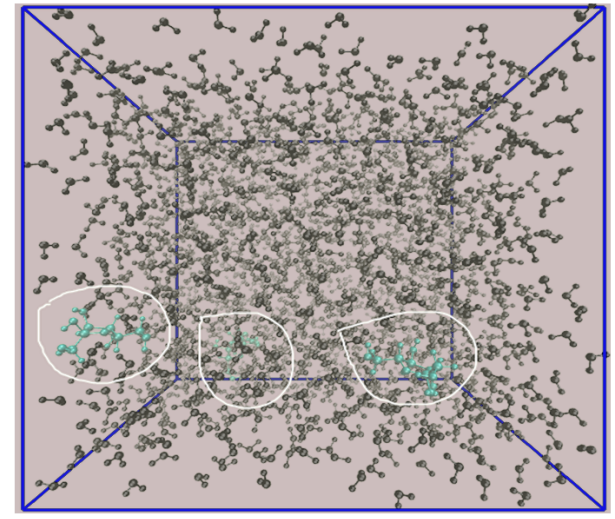

(a)

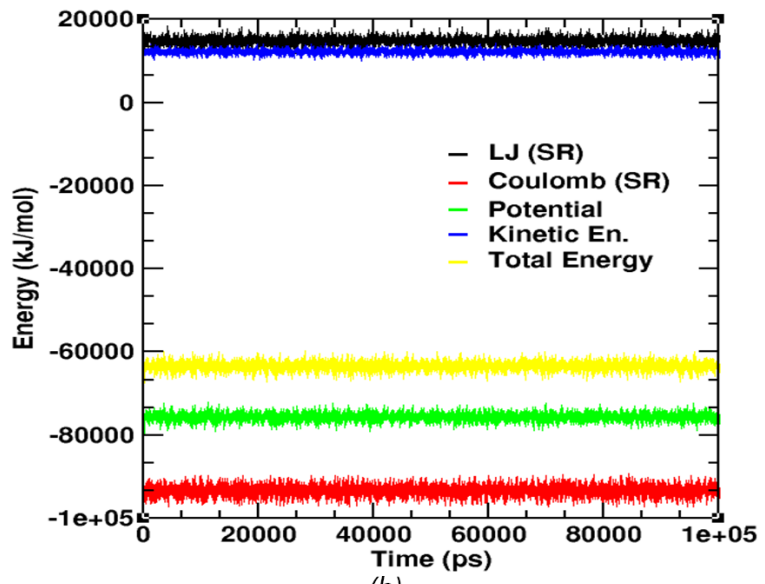

(b)

Figure 4. (a) Structure of three fructose molecules (enclosed) and water as solvent after equilibration, (b) Energy profile of the system at $\mathrm{T}=298.15 \mathrm{~K}$.

Table 3. Values of simulated temperature $\left(T_{\text {sim }}\right)$ and density at various coupling temperatures $\left(T_{F R U}\right)$.

\begin{tabular}{ccccc}
\hline S.N & $\mathrm{T}_{\text {FRU }}(\mathrm{K})$ & $\mathrm{T}_{\text {sim }}(\mathrm{K})$ & $\rho_{\text {system }}\left(\mathrm{kg} / \mathrm{m}^{3}\right)$ & $\rho_{\text {water }}\left(\mathrm{kg} / \mathrm{m}^{3}\right)[28]$ \\
\hline 1. & 298.15 & $298.11 \pm 0.037$ & $996.12 \pm 0.034$ & 997.05 \\
2. & 303.15 & $303.09 \pm 0.038$ & $993.44 \pm 0.034$ & 995.65 \\
3. & 308.15 & $308.09 \pm 0.038$ & $990.73 \pm 0.033$ & 994.03 \\
4. & 312.15 & $312.05 \pm 0.039$ & $988.39 \pm 0.033$ & 992.59 \\
\hline
\end{tabular}

The simulated values of system density are in good agreement (within maximum deviation of around 1\%) with that of water density as seen in Table 3. From Table 3, it can be inferred that the density is inversely proportional to the temperature i.e. Higher the temperature, lower is the density. After equilibration run, the production run was performed to calculate the equilibrium properties of the system such as diffusion coefficient by fixing the number of particles, volume and temperature i.e. NVT ensemble. The velocity-rescale thermostat was used for this run. We didn't couple the system to a fixed pressure and had used the structure obtained after equilibration run by which we fixed the volume of the system. The production run was carried out for $100 \mathrm{~ns}$ with the time step of 2 fs.

\section{Energy Profile}

The Fig. 4(b) represents the energy profile of the system at $298.15 \mathrm{~K}$ with the contributions of different energies. In our OPLS/AA force field, the total potential energy is the sum of Lennard-Jones and Coulomb energy. As we have used the cut-off values for Lennard-Jones and Coulomb potential, the energy corresponding to them 
are the short range energies. The total energy is the sum of potential and kinetic energies. The Lennard-Jones interaction energy is positive with an average of $14862.76 \pm 2.32 \mathrm{~kJ} \mathrm{~mol}^{-1}$. The Coulomb energy is negative with value of $-93395.37 \pm 3.78 \mathrm{~kJ} \mathrm{~mol}^{-1}$ so the potential energy, which is the sum of Lennard-Jones and Coulomb energy is negative with value of $-75665.15 \pm 2.38 \mathrm{~kJ} \mathrm{~mol}^{-1}$. The Lennard-Jones energy is positive which destabilizes the system, where as the attractive Coulomb interaction keeps the system bound and stable. This shows that the dominating part potential energy is Coulomb energy. The kinetic energy is $12250.45 \pm 1.55 \mathrm{~kJ} \mathrm{~mol}^{-1}$, so the total energy, sum of potential and kinetic energy is $-63414.70 \pm 2.87 \mathrm{~kJ} \mathrm{~mol}^{-1}$. The negative value of the total energy shows that the system is bounded and is in stable equilibrium.

\section{Results and Discussion}

In this section, we present and discuss the structural and dynamical properties with the radial distribution function (RDF), diffusivity of solute (fructose) and solvent itself, respectively, in SPC/E model of water.

\section{Radial Distribution Function}

The spherically averaged local organization around any given atom is explained by radial distribution function (RDF) and thus can be used to study structure of the system. RDF, the distribution of the neighboring molecules with respect to the reference molecule, is dimensionless function [22]. In case of periodic systems, RDF shows sharp peaks and troughs up to infinity where the separations and heights are the characteristics of the lattice structure [20]. RDF oscillates up to certain orders and then attains constant value as unity [29]. We evaluate pair distribution function in terms of RDF.

$\operatorname{RDF} \mathrm{g}(\mathbf{r})$ between oxygen atoms of water molecules $\mathrm{g}_{(\mathrm{OW}-\mathrm{OW})}(\mathbf{r})$ and oxygen $(\mathrm{O} 9)$ atom of fructose and oxygen of water molecule i.e. $\mathrm{g}_{\left(O_{9-O W}\right)}(\mathbf{r})$ has been evaluated. The value of $\mathrm{g}(\mathbf{r})$ is zero up to certain region from origin which is called exclusion region. In this region, the probability of finding particle with respect to the reference particle is zero. There is no probability of finding particle below exclusion region if it is lesser than the Van der Waals radius $\left(2^{\frac{1}{6}} \sigma_{i j}\right)$ or distance of separation is lesser than Van der Waals radius. The exclusion region is due to $\mathrm{r}^{-12}$ term of LJ interaction and repulsive coulomb interaction.

The first peak point (FPP) in RDF curve is the point where $\mathrm{g}(\mathbf{r})$ has the maximum value and corresponding value is first peak value (FPV). The oscillatory behaviour in RDF curve is reduced on going right handside in graph and finally no oscillation is achieved which implies the absence of long range correlation as $(\mathrm{g}(\mathbf{r}) \rightarrow 1[30])$. The curve of $\mathrm{g}(\mathbf{r})$ is oscillatory in nature hence particles are not distributed uniformly around the reference particle rather they are arranged in discrete spherical shell-like structures having certain radius from the central molecule. Therefore, the first peak in $\mathrm{g}(\mathbf{r})$ signifies position of the first nearest neighbor which is found in first shell and second peak indicates the position of second nearest neighbor in second shell [31]. 


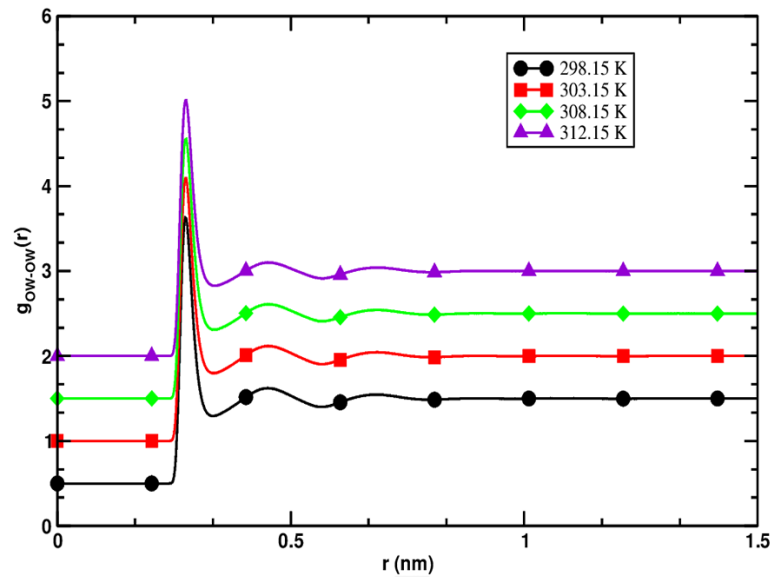

(a)

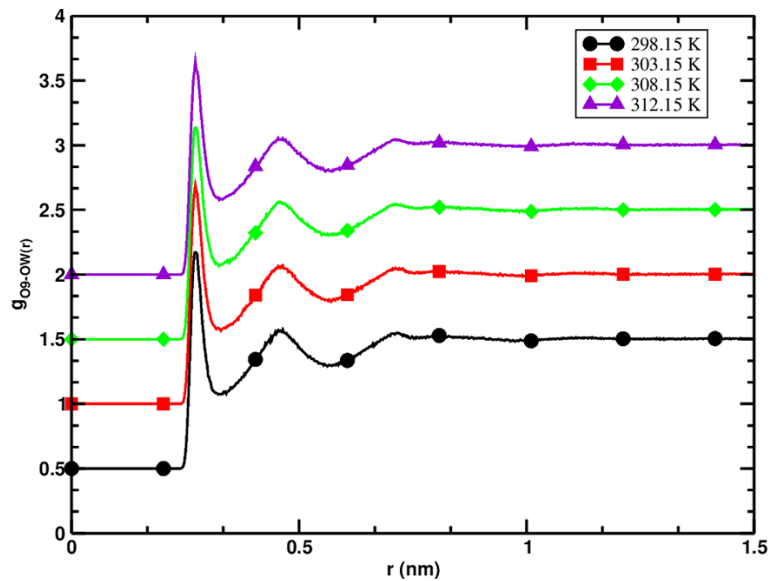

(b)

Figure 5. RDF at different temperatures. (a) Oxygen atoms of water molecules, (b) Oxygen atom of fructose molecule and oxygen atom of water molecule.

Fig. 5(a) represents the RDF of oxygen atoms of water molecules at different temperatures. It shows three clear peaks which suggest that the molecules are correlated up to third solvation shell. The value of the peak and position of the peak are provided in Table 4 .

Table 4. Simulated data for the RDF analysis between the solvent molecules.

\begin{tabular}{cccccccc}
\hline \multicolumn{7}{c}{ RDF analysis of OW-OW } \\
\hline $\mathrm{T}(\mathrm{K})$ & ER $(\mathrm{nm})$ & $\mathrm{FPP}(\mathrm{nm})$ & FPV & SPP $(\mathrm{nm})$ & SPV & TPP $(\mathrm{nm})$ & TPV \\
\hline 298.15 & 0.240 & 0.274 & 3.128 & 0.450 & 1.122 & 0.680 & 1.046 \\
303.15 & 0.240 & 0.276 & 3.092 & 0.450 & 1.118 & 0.684 & 1.044 \\
308.15 & 0.240 & 0.276 & 3.053 & 0.450 & 1.110 & 0.686 & 1.042 \\
312.15 & 0.240 & 0.276 & 3.010 & 0.456 & 1.100 & 0.690 & 1.041 \\
\hline
\end{tabular}

Note: ER - Excluded Region, FPP - First Peak Position, FPV - First Peak Value, SPP - Second Peak Position, SPV - Second Peak Value, TPP - Third Peak Position, TPV - Third Peak Value.

The equilibrium structure of the solvent i.e. water molecules is determined by the RDF of solvent. The hydrogen of water does not take part in LJ interaction in SPC/E model that we have used. Hence, gow-OW $(\mathbf{r})$ is used to study the structure of water molecule. The value of $\sigma$ for OW-OW is $0.3165 \mathrm{~nm}$, and the Van der Waals radius $\left(2^{1 / 6} \sigma\right)$ is $0.3553 \mathrm{~nm}$ [26]. From the Table 4, it is clear that the excluded region remains fairly independent of changing temperature. It also calculates that the excluded region is smaller than the Van der Waals radius and nearest neighbor separation indicating that the probability of finding correlated oxygen atoms in this region to be zero. The first peak position remains at the same position within the error of $\pm 0.002 \mathrm{~nm}$ as a function of temperature. On the contrary, SPP and TPP shift towards right on increasing temperature. The magnitudes of all the peaks decrease on rising temperature. Furthermore, the width of the peaks increases on increasing temperature. Both variations are the consequences of excess volume created in the system and the co-ordination number decreases with increase in temperature. The thermal agitation of atoms in the system 
increases with increase in temperature and solvent becomes less structural. The RDF between the oxygen of fructose and oxygen of water describes solute-solvent interaction that broadens the scope of the study is shown Fig. 5(b).

Table 5. Simulated data for the RDF analysis between oxygen (O9) atom of fructose molecule and oxygen atom of water molecule.

\begin{tabular}{lccccccc}
\hline \multicolumn{7}{c}{ RDF analysis of O9-OW } \\
\hline $\mathrm{T}(\mathrm{K})$ & ER $(\mathrm{nm})$ & FPP $(\mathrm{nm})$ & FPV & SPP $(\mathrm{nm})$ & SPV & TPP $(\mathrm{nm})$ & TPV \\
298.15 & 0.238 & 0.272 & 1.672 & 0.464 & 1.074 & 0.718 & 1.049 \\
303.15 & 0.238 & 0.272 & 1.691 & 0.464 & 1.068 & 0.720 & 1.048 \\
308.15 & 0.238 & 0.272 & 1.638 & 0.460 & 1.062 & 0.710 & 1.046 \\
312.15 & 0.238 & 0.272 & 1.646 & 0.464 & 1.054 & 0.712 & 1.046 \\
\hline
\end{tabular}

The detail of the Fig. 5(b) is given in Table (5). The three clear distinct peaks are observed in radial distribution function between the solute and solvent. The magnitude of the excluded region is independent of the change in temperature which is shown in Table 5. The correlation between solute and solvent decreases with increase in temperature which can been seen by decreasing peak value. Further, the oscillatory nature of the curve suggests that the distribution of water molecule around the reference atom isn't uniform. The roughness in the figure is due to insufficient statistics caused by the few number of fructose molecules. The value of $\sigma$ for O9-OW is $0.31175 \mathrm{~nm}$, and the van der Waals radius $\left(2^{1 / 6} \sigma\right)$ is $0.34992 \mathrm{~nm}$. The value is greater than excluded region $(0.238 \mathrm{~nm})$ and verifies the theory that there is no correlated atoms in exclusion region.

\section{Diffusion Coefficients}

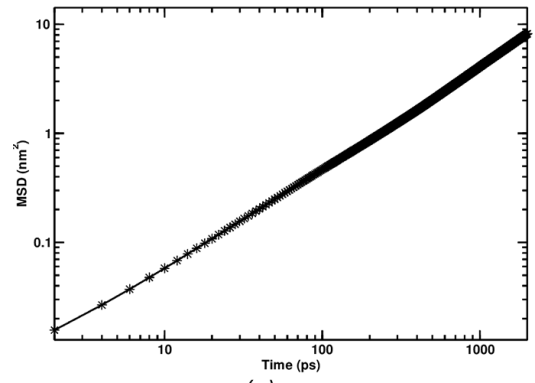

(a)

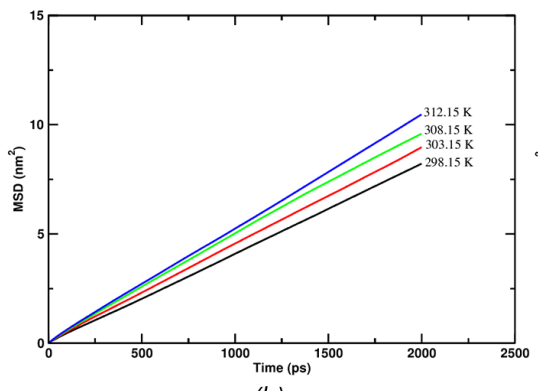

(b)

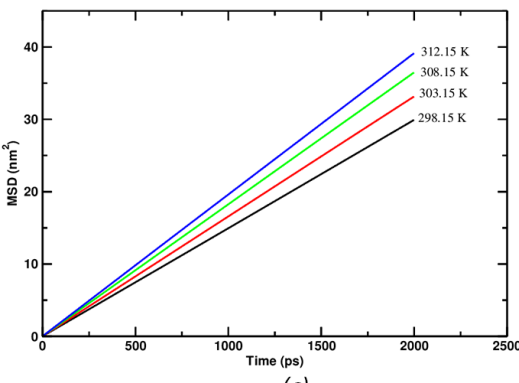

(c)

Figure 6. MSD vs time plot. (a) In logarithmic scale for fructose molecule at $298.15 \mathrm{~K}$, (b) Fructose molecule, (c) Water molecule at different temperatures.

In this section we discuss the self-diffusion coefficients of fructose and water molecules which are determined from slope of the mean square displacement (MSD) versus time (t) by using Einstein's relation shown in Eq. 1. The simulation (production run) has been performed for 100,000 ps (100 ns), however, we have taken results upto 2000 ps (2 ns) into our consideration. There is a significant difference between number of fructose and water molecules, hence, results up to 2000 ps has been considered to match our results with the case of infinite dilution. 
The high ballistic motion causes diffusion coefficient to be high at first which remains constant as time passes. This constant portion of the graph gives diffusion coefficient [22]. The ballistic region can be seen in Fig. 6(a) which is log-log plot of mean square displacement (MSD) wih time and is represented by the parabolic region of the graph very close to the origin.

The Figs. 6(b) \& 6(c) show the MSD plot of fructose and water molecules at temperatures 298.15 K, 303.15 K, 308.15 K \& $312.15 \mathrm{~K}$ respectively. The data are fitted linearly using grace and the slope of MSD curve is divided by 6 to get self-diffusion coefficient. The values of the self -diffusion coefficient of fructose and water obtained from MSD plot is presented in Tables 6-7.

Table 6. Simulated values of self-diffusion coefficient of fructose at different temperatures.

\begin{tabular}{ll}
\hline \multicolumn{2}{l}{ Self-diffusion coefficient $\left(10^{-9} \mathrm{~m}^{2} \mathrm{~s}^{-1}\right)$} \\
\hline $\mathrm{T}(\mathrm{K})$ & Simulated value \\
\hline 298.15 & $0.683 \pm 0.00012$ \\
303.15 & $0.746 \pm 0.00028$ \\
308.15 & $0.776 \pm 0.00076$ \\
312.15 & $0.863 \pm 0.00020$ \\
\hline
\end{tabular}

From both Table $6 \&$ 7, we can clearly see that self-diffusion coefficient increases with increase in temperature which is infact due to th increase in thermal agitations of molecules. The result of water molecule is compared with the experimental paper and errors are within $8.59 \%$. Thus, the self-diffusion coefficient of water obtained in our simulation is in excellent agreement with the experimental value published in various papers [32? ].

Table 7. Simulated and experimental values of self-diffusion coefficient of water at different temperatures.

\begin{tabular}{cccc}
\hline \multicolumn{4}{c}{ Self-diffusion coefficient $\left(10^{-9} \mathrm{~m}^{2} \mathrm{~s}^{-1}\right)$} \\
\hline $\mathrm{T}(\mathrm{K})$ & Simulated value & Experimental value & Error in $\%$ \\
\hline 298.15 & $2.4967 \pm 0.000088$ & $2.299[33]$ & 8.59 \\
303.15 & $2.7642 \pm 0.000046$ & $2.597[33]$ & 6.43 \\
308.15 & $3.0417 \pm 0.000029$ & $2.895[33]$ & 5.06 \\
312.15 & $3.2610 \pm 0.000064$ & $3.173[32]$ & 2.77 \\
\hline
\end{tabular}

The binary or mutual diffusion coefficient is calculated using Darken's relation (Eq. (3)) and is very close to that of self-diffusion coefficient of solute in the mixture due to lower solute concentrations. In our simulation, we have taken 1624 water molecules as solvent and 3 fructose molecules as solute which get dissolved in cubical simulation box. Hence, the mole fraction of water molecules is 0.9982 and that of fructose molecule is 0.0018 . These mole fractions along with self-diffusion coefficients are used in Darken's relation to find binary diffusion coefficients presented in Table 8. 
Table 8. Simulated and experimental values of binary diffusion coefficient of fructose in water at different temperatures.

\begin{tabular}{lccc}
\hline \multicolumn{4}{c}{ Binary diffusion coefficient (in $\left.10^{-9} \mathrm{~m}^{2} \mathrm{~s}^{-1}\right)$} \\
\hline $\mathrm{T}(\mathrm{K})$ & Simulated value & Experimental value [34] & Error in \% \\
\hline 298.15 & $0.686 \pm 0.00011$ & $0.661 \pm 0.008$ & 3.78 \\
303.15 & $0.749 \pm 0.00027$ & $0.744 \pm 0.008$ & 0.67 \\
308.15 & $0.780 \pm 0.00069$ & $0.824 \pm 0.010$ & 5.35 \\
312.15 & $0.867 \pm 0.00019$ & $0.915 \pm 0.010$ & 5.25 \\
\hline
\end{tabular}

The simulated results in this present work are found to be in harmony with the experimental results[34] and errors being within the range of $0.67 \%$ to $5.35 \%$.

\section{Temperature dependence}

The diffusion process depends strongly on temperature, being low at low temperature but high at high temperature. This temperature dependency behaviour of the diffusion can be explained by Arrhenius formula $[35]$

$$
\mathrm{D}=\mathrm{D}_{o} \exp \left(\frac{-E_{a}}{N_{A} k_{B} T}\right)
$$

Taking natural logarithm on both sides, we get

$$
E_{a}=-N_{A} \mathrm{k}_{B} \frac{\partial \ln D}{\partial(1 / T)}
$$

The intercept of the extrapolate Arrhenius line for $T^{-1} \Rightarrow 0$ yields pre-exponential factor $\left(D_{o}\right)$. Here, the slope of linear best fitted line is used for obtaining activation energy.

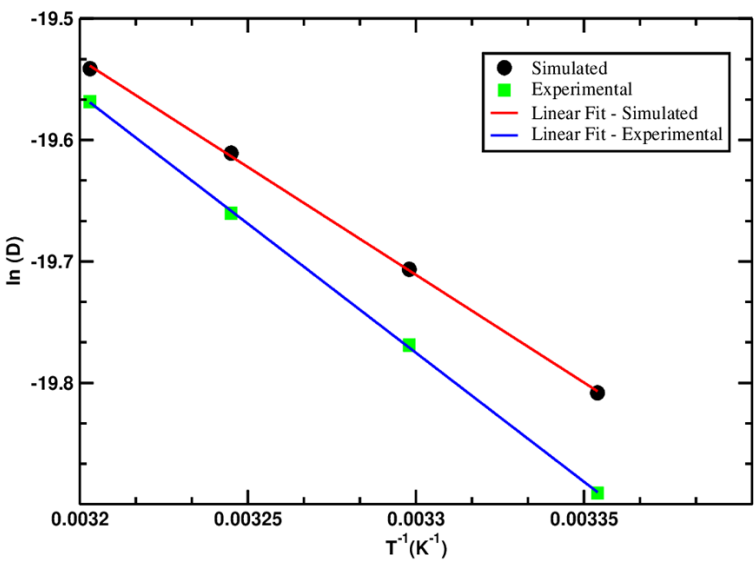

(a)

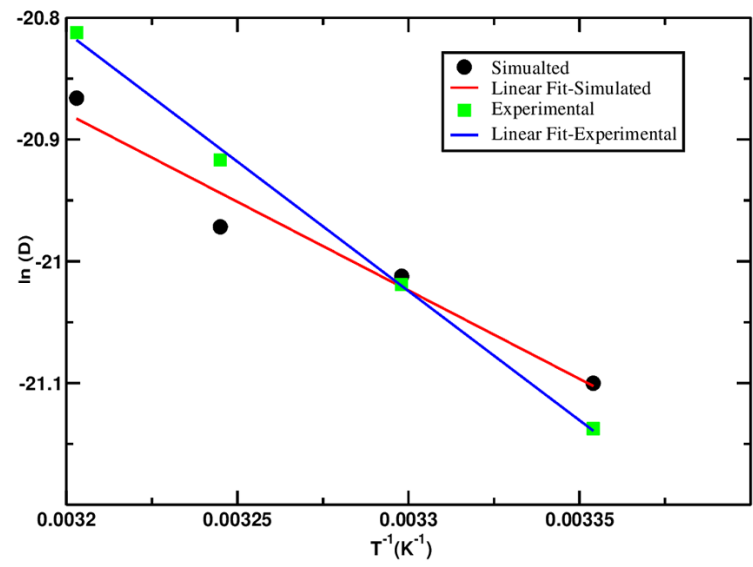

(b)

Figure 7. Arrhenius diagram of the simulated and experimental values. (a) Water, (b) Binary mixture of fructose and water. 
The temperature dependency of diffusion coefficient can be seen in Figs. 7(a) \& 7(b). The diffusion coefficient increases with increase in the temperatures. The temperature dependency of diffusion is found to follow Arrhenius behavior when simulated and experimental data are fed in Eq. 11. The Arrhenius diagram is plotted and activation energy is calculated using Eq. 12. Thus, obtained activation energies are presented in Table 9 which are found to be in excellent agreement with experimental value.

Table 9. Table for calculation of Activation Energy.

\begin{tabular}{lccc}
\hline \multicolumn{3}{c}{ Estimation of Activation Energy in $\mathrm{KJmol}^{-1}$} \\
\hline Binary mixture & Activation Energy & Water & Activation Energy \\
\hline Simulated & 12.084 & Simulated & $15.640[34]$ \\
Experimental & 14.748 & Experimental & $17.657[32 ?]$ \\
\hline
\end{tabular}

We have plotted the Arrhenius diagram and estimated the activation energy for diffusion shown in Table 9. The experimental value in the Table 9 is the value obtained by using the experimental data of diffusion coefficient for Arrhenius diagram (except for binary mixture which is the value presented in paper) and calculating activation energy from it.

\section{Conclusions}

Present work carries the Molecular Dynamics (MD) simulation to study the diffusion coefficient of binary mixture of 3 fructose molecules and 1624 water molecules at different temperatures $(298.15 \mathrm{~K}, 303.15 \mathrm{~K}, 308.15$ $\mathrm{K}$ and $312.15 \mathrm{~K}$ ). The number of molecules of fructose and water were taken in such a way that they mimic the experimental concentration $0.1 \mathrm{~mol} \mathrm{dm}^{-3}$. The self diffusion coefficients of water, and the binary diffusion coefficients of the system are found to be in excellent agreement (less than 10\%) with the experimental data available in literature. They also follow the Arrhenius Behavior. Also, the activation energy of water and the binary mixture (of water and fructose) calculated from the present work agree well with the available experimental values.

\section{References}

[1] Genova J, Zheliaskova A, Mitov M. Monosaccharides (fructose, glucose) and disaccharides (sucrose, trehalose) influence the elasticity of SOPC membranes. Journal of Optoelectronics and Advanced Materials. $2007 ; 9(2): 427$.

[2] All About Fructose; https://www.sites.google.com/site/allaboutfructose/home.

[3] Osol A, Anderson JT, Hoover JE. Remington's pharmaceutical sciences. Mack Publishing Company; 1975.

[4] Wach W. Ullmann's encyclopedia of industrial chemistry. vol. 16. Weiheim: Wiley-Vch Verlag; 2012.

[5] Lide DR. CRC handbook of chemistry and physics. vol. 79. Florida: CRC press; 1998. 
[6] Council NR. Specifications and Criteria for Biochemical Compounds. 3rd ed. Washington, DC: The National Academies Press; 1972.

[7] Sun SZ, Empie MW. Fructose metabolism in humans-what isotopic tracer studies tell us. Nutrition \& metabolism. 2012;9(1):1-15.

[8] Hendrickson K. What are the functions of fructose in the body?;. https://www.livestrong.com/article/ 279731-what-are-the-functions-of-fructose-in-the-body/.

[9] Videla E, Blanco A, Galli M, Fernandez-Collazo E. Human seminal biochemistry: fructose, ascorbic acid, citric acid, acid phosphatase and their relationship with sperm count. Andrologia. 1981;13(3):212-214.

[10] Trang NT, Huyen V, Linh N, Sang T. Seminal fructose concentration in man infertility and the fructose tests meaning in diagnosis reason of azoospermia man. Journal of Scienticfic and Technical Research. 2018;8(1):6270-6274.

[11] Bray GA. How bad is fructose? The American journal of clinical nutrition. 2007;86(4):895-896.

[12] Rippe JM, Angelopoulos TJ. Fructose-containing sugars and cardiovascular disease. Advances in Nutrition. 2015;6(4):430-439.

[13] Dinda AK, Tripathy DR, Dasgupta S. Glycation of Ribonuclease A affects its enzymatic activity and DNA binding ability. Biochimie. 2015;118:162-172.

[14] Allen M, Tildesley D. Computer simulation of liquids. USA: Oxford University Press; 1989.

[15] Rapaport DC. The art of molecular dynamics simulation. 2nd ed. Cambridge university press; 2004.

[16] Crank J. The Mathematics of Diffusion. 2nd ed. Oxford University Press; 1975.

[17] Mehrer H, Stolwijk NA. Heroes and highlights in the history of diffusion. The Open-Access Journal for the Basic Principles of Diffusion Theory, Experiment and Application. 2009;.

[18] Poudyal I, Adhikari NP. Temperature dependence of diffusion coefficient of carbon monoxide in water: A molecular dynamics study. Journal of Molecular Liquids. 2014;194:77-84.

[19] Dahal U, Adhikari NP. Molecular dynamics study of diffusion of heavy water in normal water at different temperatures. Journal of Molecular Liquids. 2012;167:34-39.

[20] Pokharel S, Aryal N, Niraula BR, Subedi A, Adhikari NP. Transport properties of methane, ethane, propane, and n-butane in water. Journal of Physics Communications. 2018;2(6):065003.

[21] Frenkel D, Smit B. Understanding molecular simulation: From algorithms to applications. 2nd ed. New York: Academic Press; 2002.

[22] Pokharel S, Pantha N, Adhikari N. Diffusion coefficients of nitric oxide in water: A molecular dynamics study. International Journal of Modern Physics B. 2016;30(27):1-20.

[23] Malenkov G. Liquid water and ices: understanding the structure and physical properties. Journal of Physics: Condensed Matter. 2009;21(28):283101.

[24] Ballenegger V, Picaud S, Toubin C. Molecular dynamics study of diffusion of formaldehyde in ice. Chemical physics letters. 2006;432(1-3):78-83. 
[25] Darken LS. Diffusion, mobility and their interrelation through free energy in binary metallic systems. Trans Aime. 1948;175:184-201.

[26] Abraham MJ and Van Der Spoel D and Lindahl E and Hess B. GROMACS user manual, version 5.0.4; 2014.

[27] Fructose Structure;. https://ligandbook.org/package/1411.

[28] Senese F. Water density calculator; 2003. https://antoine.frostburg.edu/chem/senese/javascript/ water-density.html.

[29] McQuarrie D. Statistical mechanics university science books. Harper and Row; 2000.

[30] Chandler D. Introduction to modern statistical mechanics. Oxford University Press, Oxford, UK. $1987 ; 40$.

[31] Thapa S, Adhikari N. A molecular dynamics study of oxygen gas in water at different temperatures. International Journal of Modern Physics B. 2013;27(08):1350023.

[32] Dietrich O. Diffusion coefficients of water; 2002. https://dtrx.de/od/diff/.

[33] Holz M, Heil SR, Sacco A. Temperature-dependent self-diffusion coefficients of water and six selected molecular liquids for calibration in accurate 1H NMR PFG measurements. Physical Chemistry Chemical Physics. $2000 ; 2(20): 4740-4742$.

[34] Ribeiro AC, Ortona O, Simoes SM, Santos CI, Prazeres PM, Valente AJ, et al. Binary mutual diffusion coefficients of aqueous solutions of sucrose, lactose, glucose, and fructose in the temperature range from (298.15 to 328.15) K. Journal of Chemical \& Engineering Data. 2006;51(5):1836-1840.

[35] Mehrer H. Diffusion in solids: fundamentals, methods, materials, diffusion-controlled processes. Springer Science \& Business Media; 2007. 